\title{
Adverse Birth Outcomes Among Pregnant Women With and Without COVID-19: A Comparative Study From Bangladesh
}

\section{Sumaya Binte Masud ${ }^{1,2}$, Faiza Zebeen ${ }^{1,2}$, Dil Ware Alam ', Mosharap Hossian³, Sanjana Zaman ${ }^{4}$, Rowshan Ara Begum', Mohammad Hayatun $\mathrm{Nabi}^{2}$, Mohammad Delwer Hossain Hawlader ${ }^{2}$}

'Obstetrical and Gynaecological Society of Bangladesh (OGSB), Dhaka, Bangladesh; '²Department of Public Health, North South University, Dhaka, Bangladesh; ${ }^{3}$ Public Health Professional Development Society (PPDS), Dhaka, Bangladesh; ${ }^{4}$ Department of Public Health, Daffodil International University, Dhaka, Bangladesh

Objectives: Pregnant women are especially vulnerable to respiratory infections such as coronavirus disease 2019 (COVID-19), but insufficient research has investigated pregnancy and its outcomes in women with COVID-19. This cross-sectional study compared birth outcomes related to COVID-19 between Bangladeshi pregnant women with and without COVID-19.

Methods: The study was conducted at 3 tertiary referral hospitals in Dhaka, Bangladesh, from March to August 2020. Pregnant women admitted for delivery at these hospitals with laboratory results (reverse-transcription polymerase chain reaction) were analyzed. Using convenience sampling, we included 70 COVID-19-positive and 140 COVID-19-negative pregnant women. Trained and experienced midwives conducted the interviews. Data were analyzed using the $t$-test, the chi-square test, and univariate and multivariable linear and logistic regression.

Results: Pregnant women with COVID-19 were more likely to give birth to a preterm baby (adjusted odds ratio [aOR], 2.15; 95\% confidence interval [Cl], 1.06 to 4.37 ) and undergo a cesarean section (aOR, 3.27; $95 \% \mathrm{Cl}, 1.51$ to 7.07$)$. There were no significant differences in birth weight, premature rupture of membranes, and the Apgar score at 1 minute or 5 minutes post-delivery between women with and without COVID-19. All the newborn babies who were born to COVID-19-positive women were COVID-19-negative.

Conclusions: Our study suggests that pregnant women with COVID-19 were more likely to give birth to a preterm baby and undergo a cesarean section. For this reason, physicians should be particularly cautious to minimize adverse birth outcomes among pregnant women with COVID-19 and their newborn babies.

Key words: COVID-19, Communicable diseases, Cesarean section, Preterm birth, Pregnancy outcome, Bangladesh

Received: August 6, 2021 Accepted: September 20, 2021

Corresponding author: Mohammad Delwer Hossain Hawlader Department of Public Health, North South University, Basundhara Residential Area, Dhaka 1229, Bangladesh

E-mail: mohammad.hawlader@northsouth.edu

This is an Open Access article distributed under the terms of the Creative Commons Attribution Non-Commercial License (https://creativecommons.org/licenses/by$\mathrm{nc} / 4.0 / /$ which permits unrestricted non-commercial use, distribution, and reproduction in any medium, provided the original work is properly cited.

\section{INTRODUCTION}

Coronavirus disease 2019 (COVID-19) is a highly infectious respiratory disease caused by severe acute respiratory syndrome coronavirus-2 (SARS-CoV-2) [1]. In Bangladesh, the first COVID19 positive case was detected on March 8, 2020. Bangladesh subsequently implemented extreme lockdown measures from March 26 to July 1, 2020 to limit the transmission of COVID-19 and prepare the health systems to fight the pandemic [2]. Due 
to the lockdown, the total number of institutional deliveries decreased, and the number of home deliveries escalated. This led to concerns regarding a possible increase in maternal morbidity and perinatal mortality if the situation persisted for long. In Bangladesh, during the period of COVID-19-related movement restrictions, all forms of institutional delivery (cesarean section [CS] and normal) were $15 \%$ and $20 \%$ lower in April 2020 than in April 2019 [3]. In urban areas, pregnant women went to private hospitals, and in rural areas, most pregnant women delivered their babies at home. The exact number of COVID-19-positive pregnant women in Bangladesh is unknown due to a lack of record-keeping. With limited knowledge of the effects of COVID-19 infection on pregnant women, healthcare workers faced difficulties serving such patients during this period.

Pregnant women are classified as a special demographic group due to the particular immunological state produced by pregnancy, for which reason they are more susceptible to respiratory infections such as COVID-19 and its complications $[4,5]$. Newborns are also vulnerable to several infections, but vertical transmission of COVID-19 from mother to fetus has yet to be confirmed [6]. Although a published article has reported vertical transmission, this finding has not yet been globally established [7]. A report published by the Centers for Disease Control and Prevention (CDC) in June 2020 showed that pregnant women with COVID-19 are more prone to be hospitalized with intensive care unit (ICU) support, which indicates that childbearing women are more vulnerable to have serious issues with COVID-19 [8]. A population-based study in China also suggests that COVID-19 infection during later pregnancy is linked with an increased risk of unexpected birth outcomes because of the increased number of CSs [9]. Furthermore, a case report published in September 2020 raised concern about the probable adverse outcomes of COVID-19 in women during pregnancy and suggested considering pregnant women as a group in particular danger during this pandemic [10]. The incidence of CSs, preterm births, low birth weight, neonatal ICU admission appeared to be higher among pregnant women with COVID-19 than among pregnant women without COVID-19 [11]. A study conducted with 32 hospitalized COVID-19 positive pregnant women from a COVID-19-dedicated hospital in Bangladesh revealed that $57.9 \%$ of women delivered preterm babies, and $79.0 \%$ underwent delivery by CS [12].

In contrast with the findings mentioned above, several other studies have reported no significant associations between birth outcomes and COVID-19 infection among pregnant women. A recent report analyzing 46 pregnant women with symptomatic COVID-19 found that the majority of the cases were mild, but some women developed breathing problems [13]. An observational article published in The Lancet analyzed the neonatal and obstetric findings of several hospitalized pregnant women who had contracted COVID-19 in their third trimester. The outcomes were good for all 7 mothers; none developed critical complications, and all were discharged from the hospital [14]. Another small study with COVID-19 positive pregnant women did not report the presence of the virus in cord blood, breast milk, or amniotic fluid [15]. Likewise, a study conducted among 68 COVID-19 positive pregnant women from Bangladesh concluded that COVID-19 infection during pregnancy might not be related to adverse neonatal and obstetric consequences [16]. Similar studies have been published in many countries, but were limited by small sample sizes. However, there is a dearth of literature from Bangladesh related to pregnancy and its outcomes in women with COVID-19.

Hence, this study aimed to assess the adverse birth outcomes of pregnancy among Bangladeshi women.

\section{METHODS}

\section{Study Participants and Data Collection Procedure}

This group-comparison cross-sectional study was carried out among pregnant women admitted to three tertiary level government hospitals in Dhaka, Bangladesh from March to August 2020. The hospitals were Dhaka Medical College and Hospital, Sir Salimullah Medical College and Hospital, and Mugdha Medical College and Hospital. These hospitals were chosen because they are the highest-level referral hospitals in the country and patients from all over the country are often referred to these hospitals. Midwives were deployed in the labor wards of these hospitals by the Obstetrical and Gynaecological Society of Bangladesh (OGSB) as an emergency response to combat the COVID-19 pandemic in Bangladesh. Those midwives were trained and responsible for recording pregnant women's socioeconomic and clinical data while providing services to expecting mothers. We included pregnant women who attended any of the above-mentioned tertiary hospitals for delivery purposes with COVID-19 reverse transcription polymerase chain reaction (RT-PCR) test results. Pregnant women whose COVID-19 infection status was not investigated by laboratory testing and those who were unwilling to participate in the 


\section{study were excluded.}

For every COVID-19-positive participant in the study, our experienced data collectors interviewed two COVID-19-negative pregnant women from the same facility to increase the sample size for comparing outcomes. For data collection, we used nonprobability convenience sampling. In total, we screened 110 COVID-19-positive pregnant women treated at those hospitals during the study period for inclusion, of whom 70 remained after applying the inclusion and exclusion criteria. Corresponding to the 70 COVID-19-positive pregnant women, we included 140 COVID-19-negative pregnant women admitted to the abovementioned hospitals for delivery during the study period.

We collected data on participants' socio-demographic information of the participants, the characteristics of pregnant women, and the birth outcomes of newborns.

\section{Quality Control}

The supervisors regularly inspected the dependability and competency of the completed questionnaires throughout the data collection period. Simultaneously, the data entry team carefully entered and cleaned the collected data to reduce the likelihood of missing or irrelevant data.

\section{Statistical Analysis}

Data were analyzed using Stata version 15.0 (StataCorp., College Station, TX, USA). Continuous variables were expressed as mean with standard deviation, whereas categorical variables were expressed as frequency and percentages (\%). In univariate analyses, we conducted the $t$-test for continuous outcomes, and the chi-square test was performed for categorical outcomes. As appropriate, we investigated associations between explanatory variables and outcome variables using a multivariable binary logistic regression model, a Firth logistic regression model, and a multivariable linear regression model. Firth logistic regression is especially useful for minimizing the bias caused by a small sample and overcoming the complete separation of predictor variables over the outcome variable of a multivariable logistic regression model [17]. The results were reported as adjusted odds ratios (aORs) and the corresponding $95 \%$ confidence intervals ( $\mathrm{Cls}$ ). We considered $p$-values $<0.05$ as indicating statistical significance.

\section{Ethics Statement}

The study was conducted according to the guidelines stated in the Declaration of Helsinki and ethical approval was obtained from the Institutional Review Board of North South University (2020/OR-NSU/IRB-No. 0801). Participants were thoroughly informed regarding the purpose of the study and written informed consent was obtained from participants before data collection.

\section{RESULTS}

Table 1 presents the respondents' characteristics. We found that the COVID-19-positive women were older than the COVID19-negative women, and there was a significant relationship between COVID-19 status and age groups $(p<0.001)$. The proportion of employed women was slightly higher in the COVID19-infected group (22.9\%) than those who were not infected with COVID-19 (20.0\%). In the non-infected group, $40.7 \%$ of respondents were pregnant for the first time, which was slightly

Table 1. Associations between respondents' characteristics and coronavirus disease 2019 (COVID-19) status among Bangladeshi pregnant women

\begin{tabular}{|c|c|c|c|}
\hline \multirow{2}{*}{ Characteristics } & \multicolumn{2}{|c|}{ COVID-19 infection status } & \multirow{2}{*}{$p$-value } \\
\hline & Infected & Non-infected & \\
\hline \multicolumn{3}{|l|}{ Age (y) } & $<0.001$ \\
\hline$\leq 20$ & $6(8.6)$ & $53(37.9)$ & \\
\hline $21-25$ & $19(27.1)$ & $44(31.4)$ & \\
\hline $26-30$ & $24(34.3)$ & $33(23.6)$ & \\
\hline$>30$ & $21(30.0)$ & $10(7.1)$ & \\
\hline \multicolumn{3}{|l|}{ Occupation } & 0.632 \\
\hline Homemaker & $54(77.1)$ & $112(80.0)$ & \\
\hline Employed & $16(22.9)$ & $28(20.0)$ & \\
\hline \multicolumn{3}{|l|}{ Gravidity } & 0.676 \\
\hline 1 & $25(35.7)$ & $57(40.7)$ & \\
\hline 2 & $20(28.6)$ & $41(29.3)$ & \\
\hline$\geq 3$ & $25(35.7)$ & $42(30.0)$ & \\
\hline \multicolumn{3}{|l|}{ Parity } & 0.133 \\
\hline 1 & $26(37.1)$ & $63(45.0)$ & \\
\hline 2 & $16(22.9)$ & $40(28.6)$ & \\
\hline$\geq 3$ & $28(40.0)$ & $37(26.4)$ & \\
\hline \multicolumn{3}{|c|}{$\begin{array}{l}\text { Pregnancy complications (antepartum } \\
\text { hemorrhage, eclampsia, etc.) }\end{array}$} & 0.328 \\
\hline No & 67 (95.7) & $129(92.1)$ & \\
\hline Yes & $3(4.3)$ & $11(7.9)$ & \\
\hline \multicolumn{3}{|c|}{$\begin{array}{l}\text { Chronic disease (hypertension, asthma, diabetes, } \\
\text { hypothyroidism, hepatitis B infection, etc.) }\end{array}$} & 0.004 \\
\hline No & $60(85.7)$ & $135(96.4)$ & \\
\hline Yes & $10(14.3)$ & $5(3.6)$ & \\
\hline
\end{tabular}

Values are presented as number (\%). 
higher than the corresponding proportion in the infected group (35.7\%). However, $40.0 \%$ of the COVID-19-positive women had a parity of 3 or more, whereas this proportion was much lower among women without COVID-19 (26.4\%). Pregnancy-related complications were also slightly more common in the nonCOVID-19 group than in the COVID-10 group (7.9 vs. $4.3 \%)$. In contrast, a higher proportion of participants with COVID-19 (14.3\%) reported comorbidities than participants without COVID-19 (3.6\%), and the presence of at least 1 comorbidity was statistically significantly associated with participants' COVID19 infection status $(p=0.004)$.

Table 2 shows the association between birth outcomes and COVID-19 infection status among Bangladeshi pregnant women. CSs were much more common (71.4\%) among the COVID19-positive women than among their COVID-19-negative coun-

Table 2. Associations between birth outcomes and the coronavirus disease 2019 (COVID-19) infection status of Bangladeshi pregnant women

\begin{tabular}{|c|c|c|c|}
\hline \multirow{2}{*}{ Variables } & \multicolumn{2}{|c|}{ COVID-19 infection status } & \multirow{2}{*}{$p$-value } \\
\hline & Infected & Non-infected & \\
\hline Premature rupture of membranes & & & 0.156 \\
\hline No & $69(98.6)$ & $140(100.0)$ & \\
\hline Yes & $1(1.4)$ & $0(0.0)$ & \\
\hline Gestational age (wk) & & & 0.001 \\
\hline Preterm (<37) & 37 (52.9) & $42(30.0)$ & \\
\hline Term $(\geq 37)$ & $33(47.1)$ & $98(70.0)$ & \\
\hline Mode of delivery & & & $<0.001$ \\
\hline NVD & $20(28.6)$ & $81(57.9)$ & \\
\hline CS & $50(71.4)$ & $59(42.1)$ & \\
\hline Status of baby & & & 0.125 \\
\hline Alive & $66(94.3)$ & $137(97.9)$ & \\
\hline Stillborn & $2(2.9)$ & $0(0.0)$ & \\
\hline IUD & $2(2.9)$ & $3(2.1)$ & \\
\hline Birth weight of baby (kg) & & & 0.399 \\
\hline Low birth weight $(<2.5)$ & $17(25.0)$ & $27(19.9)$ & \\
\hline Normal birth weight $(\geq 2.5$ ) & $51(75.0)$ & $109(80.2)$ & \\
\hline Sex of baby & & & 0.674 \\
\hline Male & $38(54.3)$ & $70(50.0)$ & \\
\hline Female & $32(45.7)$ & $70(50.0)$ & \\
\hline COVID-19 status of the newborn & & & - \\
\hline Positive & $0(0.0)$ & $0(0.0)$ & \\
\hline Negative & $66(100)$ & $137(100)$ & \\
\hline Apgar score 1 min after delivery & $7.40 \pm 1.88$ & $7.79 \pm 1.33$ & 0.088 \\
\hline Apgar score 5 min after delivery & $9.09 \pm 2.32$ & $9.50 \pm 1.51$ & 0.125 \\
\hline
\end{tabular}

Values are presented as number (\%) or mean \pm standard deviation. NVD, normal vaginal delivery; CS, cesarean section; IUD, intrauterine death. terparts (42.1\%), and the mode of delivery was statistically significantly associated with the COVID-19 status of the participants $(p<0.001)$. Stillbirth and intrauterine death $(5.8 \%)$ were slightly more common among the COVID-19-positive women than among the COVID-19-negative women (2.1\%). The majority (52.9\%) of the COVID-19-positive women had premature delivery, whereas this was only the case for $30.0 \%$ of the COVID-19-negative women, and the infection status of the respondents was found to be significantly associated with the gestational age $(p=0.001)$. A slight difference was found in the likelihood of giving birth to a normal-weight infant between COVID-19-positive (75.0\%) and COVID-19-negative women (80.2\%). The Apgar score is widely used to evaluate newborns' conditions, and we recorded it at 1 minute and 5 minutes after birth. The 1-minute Apgar score was higher in the COVID-19-negative group (7.79 \pm 1.33 ) than in the COVID-19-positive group

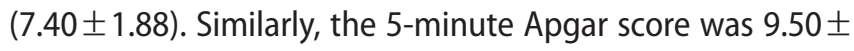
1.51 in COVID-19-negative women, which was slightly higher than in COVID-19-positive women (9.09 \pm 2.32$)$. Neither of the Apgar scores was statistically significantly associated with pregnant women's COVID-19 infection status. Likewise, premature rupture of membranes and the baby's sex were not significantly associated with mothers' COVID-19 infection status. Another important finding is that all alive newborn babies were COVID-19-negative on RT-PCR tests.

Table 3 shows the associations between the COVID-19 status of the participants and adverse birth outcomes of the newborns (preterm birth, CS delivery, and low birth weight). Multivariable logistic regression showed that, compared to women without COVID-19 infection, COVID-19-positive women were 2.15 times ( $95 \% \mathrm{Cl}, 1.06$ to 4.37$)$ more likely to give birth to a preterm baby.

We performed Firth logistic regression modeling to assess

Table 3. Results of the multivariable logistic regression model for associations between maternal coronavirus disease 2019 (COVID-19) status and adverse birth outcomes of the newborns (preterm birth, cesarean section delivery, and low birth weight) ${ }^{1}$

\begin{tabular}{lccc}
\hline $\begin{array}{c}\text { COVID-19 } \\
\text { status }\end{array}$ & $\begin{array}{c}\text { Preterm } \\
\text { birth }\end{array}$ & $\begin{array}{c}\text { Cesarean } \\
\text { section }\end{array}$ & $\begin{array}{c}\text { Low birth } \\
\text { weight }\end{array}$ \\
\hline Non-infected & 1.00 (reference) & 1.00 (reference) & 1.00 (reference) \\
Infected & $2.15(1.06,4.37)$ & $3.27(1.51,7.07)$ & $1.56(0.64,3.80)$ \\
\hline
\end{tabular}

Values are presented as adjusted odds ratio ( $95 \%$ confidence interval). ${ }^{1}$ Adjusted for maternal age ( $\left.\leq 20,21-25,26-30,>30\right)$, occupation (employed, homemaker), gravidity $(1,2, \geq 3)$, parity $(1,2, \geq 3)$, pregnancy complications (yes, no), and chronic disease (yes, no). 
Table 4. Results of the multivariable linear regression model for the association between maternal coronavirus disease 2019 (COVID-19) status and newborns' Apgar scores

\begin{tabular}{|c|c|c|c|c|}
\hline \multirow{2}{*}{ COVID-19 status } & \multicolumn{2}{|c|}{ Apgar score at $1 \mathrm{~min}$} & \multicolumn{2}{|c|}{ Apgar score after $5 \mathrm{~min}$} \\
\hline & Unadjusted & Adjusted $^{1}$ & Unadjusted & Adjusted $^{1}$ \\
\hline Non-infected & 1.00 (reference) & 1.00 (reference) & 1.00 (reference) & 1.00 (reference) \\
\hline Infected & $-0.39(-0.83,0.06)$ & $-0.40(-0.90,0.10)$ & $-0.41(-0.94,0.11)$ & $-0.44(-1.04,0.15)$ \\
\hline
\end{tabular}

Values are presented as coefficient $(95 \%$ confidence interval).

${ }^{1}$ Adjusted for maternal age $(\leq 20,21-25,26-30,>30)$, occupation (employed, homemaker), gravidity $(1,2, \geq 3)$, parity $(1,2, \geq 3)$, pregnancy complications (yes, no), and chronic disease (yes, no).

the effect of the mother's COVID-19 status on the mode of delivery. As the dichotomous outcome variable was completely separated over a predictor variable (e.g., disease status), traditional maximum likelihood regression might not have been the best choice here. For this reason, we applied Firth logistic regression to minimize the bias caused by a small sample and to overcome the issue of complete separation for a multivariable logistic model (Table 3).

After adjusting for covariates, women with COVID-19 infection were 3.27 times $(95 \% \mathrm{Cl}, 1.51$ to 7.07$)$ more likely to undergo a CS than women without COVID-19 infection.

The multivariable logistic regression model also showed no significant association (aOR, 1.56; $95 \% \mathrm{Cl}, 0.64$ to 3.80$)$ between newborns' low birth weight and the pregnant women's COVID-19 status.

We conducted multivariable linear regression to investigate the association between newborns' Apgar scores and pregnant women's COVID-19 status. No significant associations were found for the 1-minute Apgar score (adjusted coefficient, -0.40 ; $95 \% \mathrm{Cl},-0.90$ to 0.10 ) or the 5-minute Apgar score (adjusted coefficient, $-0.44 ; 95 \% \mathrm{Cl},-1.04$ to 0.15 ) (Table 4).

\section{DISCUSSION}

Using data from 210 pregnant women and their newborn babies in Bangladesh, we investigated how COVID-19 infection affects birth outcomes. Our analysis indicated that COVID-19infected pregnant women had a higher risk of undergoing a CS delivery and giving birth to preterm babies than pregnant women who were not infected with COVID-19. Another interesting finding is that we did not find any evidence of vertical transmission of COVID-19 from pregnant women to their children. Our study results also revealed no significant relationship between birth weight and pregnant women's COVID-19 status. Furthermore, we did not find a statistically significant relationship between Apgar scores and the COVID-19 status of pregnant women.

Our study findings suggest that COVID-19 infection was more prevalent among older pregnant women than among younger ones. Several studies conducted in various parts of the world among the general population have also found results concordant with our study $[18,19]$. According to the World Health Organization, pregnant women who are older, obese, or have comorbidities such as high blood pressure and diabetes mellitus are at critical risk of severe COVID-19 outcomes [20]. The CDC also reported that pregnant women aged 35-44 years with COVID-19 infection were about 4 times more likely to require invasive ventilation and 2 times more likely to die than non-pregnant women of the same age group [21]. Blagosklonny [22] explained how vulnerability to COVID-19 is associated with age through the hyperfunction theory of quasi-programmed aging. We know that aging is primarily related to increased cellular and systemic functions (hyperfunction), which results in age-related diseases. Hypertension, for example, is a systemic hyperfunction caused by excessive activity of numerous cell types, including arterial smooth muscle cells. Likewise, COVID19 vulnerability is related to the hyperfunction of various inflammatory cells, which cause hypercoagulation, a cytokine storm, and dysfunction of the lung and distant organs [22]. A study conducted by a Japanese health ministry research team found that COVID-19-infected pregnant women of age 30 or older faced a 17\% greater risk of becoming seriously ill [23]. Furthermore, Weng et al. [24] reported an association between pregnancy at an advanced age with a heightened risk of unexpected birth outcomes, such as CS delivery and premature birth. The similar results of our study regarding adverse birth outcomes among COVID-19-positive women and the presence of a higher proportion of comparatively older women among COVID-19-infected participants solidify the findings of Weng et al. [24]. 
In this study, CS deliveries were more common (71.4\%) among COVID-19-positive pregnant women than among COVID-19-negative women (42.1\%). Along with the usual cases of CS deliveries, the physicians conducted cesarean sections as required when COVID-19-related severe complications developed among pregnant women or their fetuses (e.g., maternal breathing issues or fetal distress). This accounts for the higher rate of CS deliveries among the COVID-19-infected pregnant women than among their counterparts. A systematic review including 108 pregnancies reported that $91 \%$ of the infants born to COVID-19 positive women were delivered by CS, which is in line with our findings of a higher prevalence of CS deliveries among COVID-19-positive pregnant women [15]. Another brief review conducted by Parazzini et al. [25] reported that vaginal delivery was disproportionately less common among COVID-19-positive women. Furthermore, studies performed on Chinese [26], Indian [27], Iranian [28], and United Kingdom [29] COVID-19infected pregnant women also reported similar results.

In the present study, we observed a higher rate of premature delivery among COVID-19-positive pregnant women; the odds of giving birth to preterm babies were 2 times higher among COVID-19-positive women than among those who were COVID-19-free during delivery. Similar to our findings, a recent rapid review indicated that premature delivery affected almost half of women (47\%) hospitalized with COVID-19 infection [30]. Along with this, a prospective prepared by Schwartz and Graham [31] outlined that the previous severe acute respiratory syndrome and Middle East respiratory syndrome viruses were associated with premature birth, ICU support for newborn babies, and ultimately death in some cases. From the existing literature, we know that immune responses cause inflammation in the body [32], which is considered a significant risk factor for premature labor [33]. In cases of COVID-19 infection, all the components of the immune system appear to be involved in the immune response to reduce viral load and promote early recovery from the illness [34]. This explains why pregnant women with COVID-19 are more likely to give birth to preterm babies.

We found a non-significant association between newborns' low birth weight and the COVID-19 status of pregnant women (Table 3). In line with our findings, a systematic review and meta-analysis conducted by Melo and Araújo [35] found no significant association between the COVID-19 status of pregnant women and the birth weight of newborns. A retrospective comparative study conducted in Hubei, China, also re- ported no significant difference in newborn birth weight between pregnant women with and without COVID-19 [36].

According to our study, the prevalence of intrauterine death/ stillbirth was higher among participants with COVID-19 than among their counterparts, but the difference was not statistically significant. A study conducted using the Global Pregnancy and Neonatal outcomes in COVID-19 and American Academy of Pediatrics Section on Neonatal-Perinatal Medicine registries also reported that COVID-19 in pregnancy was not significantly associated with stillbirth or intrauterine death [37].

Our study results showed that all newborns, including babies born to COVID-19-positive pregnant women, were RT-PCRconfirmed to be COVID-19 negative. This positive birth outcome regarding the vertical transmission of COVID-19 aligns with those reported in several previous studies [7,9,35,38].

Although the prevalence of pregnant women with comorbidities was higher in the COVID-19-positive group than in the COVID-19-negative group, all pregnant women with comorbidities underwent CS deliveries. A study conducted in the United States also found that the CS delivery rate was 2.66 times higher among pregnant women with comorbidities than among those who did not have reported comorbidities [39].

To the best of our knowledge, our study is the first-ever instance of recording babies' vital signs at birth to compare the Apgar scores of babies born to women with and without COVID-19 infection. The mean Apgar score at the first minute was lower (7.40 \pm 1.88 ) among COVID-19-positive women than among COVID-19-negative women (7.79 \pm 1.33 ), but we did not find any significant relationship between the 1-minute or 5-minute Apgar scores and the pregnant women (Table 4).

\section{Strengths and Limitations}

The main strength of our study is that it included a comparison group of COVID-19-negative pregnant women. Moreover, the involvement of trained midwives in interviews and clinical assessments provided a new dimension to this study. Along with its strengths, this study has several limitations that we should mention here. Like all other cross-sectional studies, the nature of our study design did not allow us to establish a causeeffect relationship between the predictor variables and outcomes. Although we recruited samples from Bangladesh's top tertiary referral hospitals, the relatively small sample size and the unmatched comparison group are the largest obstacles to generalizing the results. Furthermore, due to movement restrictions during the pandemic, we could not reach peripheral 
hospitals, meaning that a significant proportion of eligible participants remained beyond the study's scope.

In conclusion, we found a higher chance of CS delivery and premature birth among COVID-19-positive women. In addition, we observed that the COVID-19-positive group included a high prevalence of older mothers, who are more vulnerable to develop COVID-19-related complications. Our study suggests that pregnant women and physicians should follow all COVID-19 infection-related precautions to minimize adverse birth outcomes. Attending midwives and physicians should also provide additional care to the newborn children of the COVID-19 positive mothers.

\section{CONFLICT OF INTEREST}

The authors have no conflicts of interest associated with the material presented in this paper.

\section{FUNDING}

None.

\section{ACKNOWLEDGEMENTS}

We would like to express our heartfelt appreciation and gratitude to the hospital authorities for their kind support and assistance during the research process. We are thankful to the patients for their patience and cooperation. We are also thankful to the midwives for their fantastic efforts in data collection, and to OGSB \& North South University (NSU) for their cooperation.

\section{AUTHOR CONTRIBUTIONS}

Conceptualization: SBM, MDHH. Data curation: FZ. Formal analysis: MDHH, DWA, MH. Funding acquisition: N/A. Methodology: MHN, RAB. Project administration: SBM. Visualization: MHN, SZ. Writing - original draft: SBM, FZ, DWA. Writing - review \& editing: $\mathrm{MDHH}, \mathrm{SZ}, \mathrm{RAB}, \mathrm{MH}, \mathrm{MHN}$.

\section{ORCID}

Sumaya Binte Masud https://orcid.org/0000-0001-82284796

Faiza Zebeen https://orcid.org/0000-0002-4505-434X

Dil Ware Alam https://orcid.org/0000-0003-1794-705X
Mosharop Hossian $\quad$ https://orcid.org/0000-0001-9584-7076

Sanjana Zaman https://orcid.org/0000-0002-8856-2352

Rowshan Ara Begum https://orcid.org/0000-0002-9191-5948

Mohammad Hayatun Nabi https://orcid.org/0000-0001-98162080

Mohammad Delwer Hossain Hawlader https://orcid.org/ 0000-0002-1443-6257

\section{REFERENCES}

1. Qiao J. What are the risks of COVID-19 infection in pregnant women? Lancet 2020;395(10226):760-762.

2. Sun M, Xu G, Yang Y, Tao Y, Pian-Smith M, Madhavan V, et al. Evidence of mother-to-newborn infection with COVID-19. Br J Anaesth 2020;125(2):e245-e247.

3. Ainul S, Hossain M, Bhuiyan M, Hossain SM, Rob U, Bajracharya A. Trends in maternal health services in Bangladesh before, during and after COVID-19 lockdowns: evidence from national routine service data; 2020 [cited 2021 Aug 5]. Available from: https://knowledgecommons.popcouncil.org/cgi/viewcontent. cgi?article $=2299 \&$ context $=$ departments_sbsr-rh .

4. Mor G, Cardenas I. The immune system in pregnancy: a unique complexity. Am J Reprod Immunol 2010;63(6):425-433.

5. Liu H, Wang LL, Zhao SJ, Kwak-Kim J, Mor G, Liao AH. Why are pregnant women susceptible to COVID-19? An immunological viewpoint. J Reprod Immunol 2020;139:103122.

6. Marim F, Karadogan D, Eyuboglu TS, Emiralioglu N, Gurkan CG, Toreyin ZN, et al. Lessons learned so far from the pandemic: a review on pregnants and neonates with COVID-19. Eurasian J Med 2020;52(2):202-210.

7. Fenizia C, Biasin M, Cetin I, Vergani P, Mileto D, Spinillo A, et al. Analysis of SARS-CoV-2 vertical transmission during pregnancy. Nat Commun 2020;11(1):5128.

8. Wong SF, Chow KM, Leung TN, Ng WF, Ng TK, Shek CC, et al. Pregnancy and perinatal outcomes of women with severe acute respiratory syndrome. Am J Obstet Gynecol 2004;191(1): 292-297.

9. Yang R, Mei H, Zheng T, Fu Q, Zhang Y, Buka S, et al. Pregnant women with COVID-19 and risk of adverse birth outcomes and maternal-fetal vertical transmission: a population-based cohort study in Wuhan, China. BMC Med 2020;18(1):330.

10. Ronnje L, Länsberg JK, Vikhareva O, Hansson SR, Herbst A, Zaigham M. Complicated COVID-19 in pregnancy: a case report with severe liver and coagulation dysfunction promptly improved by delivery. BMC Pregnancy Childbirth 2020;20(1): 
511.

11. Smith V, Seo D, Warty R, Payne O, Salih M, Chin KL, et al. Maternal and neonatal outcomes associated with COVID-19 infection: a systematic review. PLoS One 2020;15(6):e0234187.

12. Mustafa FN, Yasmin N, Islam M, Mehzabin S, Siddique S, Alam S. Pregnancy outcome in covid-19 positive women in a covid dedicated hospital. J Dhaka Med Coll 2020;29(2):153-157.

13. Kontou PI, Braliou GG, Dimou NL, Nikolopoulos G, Bagos PG. Antibody tests in detecting SARS-CoV-2 infection: a meta-analysis. Diagnostics (Basel) 2020;10(5):319.

14. Chen Y, Peng H, Wang L, Zhao Y, Zeng L, Gao H, et al. Infants born to mothers with a new coronavirus (COVID-19). Front Pediatr 2020;8:104.

15. Zaigham M, Andersson O. Maternal and perinatal outcomes with COVID-19: a systematic review of 108 pregnancies. Acta Obstet Gynecol Scand 2020;99(7):823-829.

16. Sayeed SJ, Rahman MM, Kabir AH, Moniruzzaman M, Mahmud $\mathrm{R}$, Yusuf MA, et al. Clinical, laboratory characteristics and pregnancy outcome of COVID-19 patients admitted in the largest COVID dedicated hospital of Bangladesh. Am J Intern Med 2021;9(1):11-16.

17. Karabon P. Rare events or non-convergence with a binary outcome? The power of Firth regression in PROC LOGISTIC; 2020 [cited 2021 Aug 4]. Available from: https://www.sas.com/content/dam/SAS/support/en/sas-global-forum-proceedings/ 2020/4654-2020.pdf.

18. Zhou F, Yu T, Du R, Fan G, Liu Y, Liu Z, et al. Clinical course and risk factors for mortality of adult inpatients with COVID-19 in Wuhan, China: a retrospective cohort study. Lancet 2020;395 (10229):1054-1062.

19. Wu C, Chen X, Cai Y, Xia J, Zhou X, Xu S, et al. Risk factors associated with acute respiratory distress syndrome and death in patients with coronavirus disease 2019 pneumonia in Wuhan, China. JAMA Intern Med 2020;180(7):934-943.

20. World Health Organization. Coronavirus disease (COVID-19): pregnancy and childbirth; 2021 [cited 2021 Sep 9]. Available from: https://www.who.int/news-room/questions-and-answers/item/coronavirus-disease-covid-19-pregnancy-andchildbirth.

21. Zambrano LD, Ellington S, Strid P, Galang RR, Oduyebo T, Tong $\mathrm{VT}$, et al. Update: characteristics of symptomatic women of reproductive age with laboratory-confirmed SARS-CoV-2 infection by pregnancy status - United States, January 22-October 3, 2020. MMWR Morb Mortal Wkly Rep 2020;69(44):1641-1647. 22. Blagosklonny MV. From causes of aging to death from COVID-
19. Aging (Albany NY) 2020;12(11):10004-10021.

23. Women in late stages of pregnancy at higher COVID-19 risk. Japan Times; 2021 Aug 22 [cited 2021 Sep 9]. Available from: https://www.japantimes.co.jp/news/2021/08/22/national/ pregnant-women-coronavirus/.

24. Weng YH, Yang CY, Chiu YW. Risk assessment of adverse birth outcomes in relation to maternal age. PLoS One 2014;9(12): e114843.

25. Parazzini F, Bortolus R, Mauri PA, Favilli A, Gerli S, Ferrazzi E. Delivery in pregnant women infected with SARS-CoV-2: a fast review. Int J Gynaecol Obstet 2020;150(1):41-46.

26. Chen L, Li Q, Zheng D, Jiang H, Wei Y, Zou L, et al. Clinical characteristics of pregnant women with Covid-19 in Wuhan, China. N Engl J Med 2020;382(25):e100.

27. Gupta P, Kumar S, Sharma SS. SARS-CoV-2 prevalence and maternal-perinatal outcomes among pregnant women admitted for delivery: experience from COVID-19-dedicated maternity hospital in Jammu, Jammu and Kashmir (India). J Med Virol 2021;93(9):5505-5514.

28. Vizheh M, Muhidin S, Aghajani F, Maleki Z, Bagheri F, Hosamirudsari $\mathrm{H}$, et al. Characteristics and outcomes of COVID-19 pneumonia in pregnancy compared with infected nonpregnant women. Int J Gynaecol Obstet 2021;153(3):462-468.

29. Knight M, Bunch K, Vousden N, Morris E, Simpson N, Gale C, et al. Characteristics and outcomes of pregnant women admitted to hospital with confirmed SARS-CoV-2 infection in UK: national population based cohort study. BMJ 2020;369:m2107.

30. Mullins E, Evans D, Viner RM, O'Brien P, Morris E. Coronavirus in pregnancy and delivery: rapid review. Ultrasound Obstet Gynecol 2020;55(5):586-592.

31. Schwartz DA, Graham AL. Potential maternal and infant outcomes from (Wuhan) coronavirus 2019-nCoV infecting pregnant women: lessons from SARS, MERS, and other human coronavirus infections. Viruses 2020;12(2):194.

32. Chen L, Deng H, Cui H, Fang J, Zuo Z, Deng J, et al. Inflammatory responses and inflammation-associated diseases in organs. Oncotarget 2017;9(6):7204-7218.

33. Hantoushzadeh S, Anvari Aliabad R, Norooznezhad AH. Antibiotics, inflammation, and preterm labor: a missed conclusion. J Inflamm Res 2020;13:245-254.

34. García LF. Immune response, inflammation, and the clinical spectrum of COVID-19. Front Immunol 2020;11:1441.

35. Melo GC, Araújo KC. COVID-19 infection in pregnant women, preterm delivery, birth weight, and vertical transmission: a systematic review and meta-analysis. Cad Saude Publica 2020; 
36(7):e00087320.

36. Zhang L, Jiang Y, Wei M, Cheng BH, Zhou XC, Li J, et al. Analysis of the pregnancy outcomes in pregnant women with COVID19 in Hubei Province. Zhonghua Fu Chan Ke Za Zhi 2020;55(3): 166-171 (Chinese).

37. Mullins E, Hudak ML, Banerjee J, Getzlaff T, Townson J, Barnette K, et al. Pregnancy and neonatal outcomes of COVID-19: core- porting of common outcomes from PAN-COVID and AAP-SONPM registries. Ultrasound Obstet Gynecol 2021;57(4):573-581.

38. Moreno SC, To J, Chun H, Ngai IM. Vertical transmission of COVID-19 to the neonate. Infect Dis Obstet Gynecol 2020;2020: 8460672.

39. Suidan RS, Apuzzio JJ, Williams SF. Obesity, comorbidities, and the cesarean delivery rate. Am J Perinatol 2012;29(8):623-628. 